\title{
On the Reality of the Continuum Discussion Note: A Reply to Ormell, 'Russell's Moment of Candour', Philosophy
}

\author{
ANNE NEWSTEAD AND JAMES FRANKLIN
}

\section{Introduction}

In a recent article ('The Continuum: Russell's Moment of Candour'), Christopher Ormell argues against the traditional mathematical view that the real numbers form an uncountably infinite set. ${ }^{1}$ He rejects the conclusion of Cantor's diagonal argument for the higher, non-denumerable infinity of the real numbers. He does so on the basis that the classical conception of a real number is mysterious, ineffable, and epistemically suspect. Instead, he urges that mathematics should admit only 'well-defined' real numbers as proper objects of study. In practice, this means excluding as inadmissible all those real numbers whose decimal expansions cannot be calculated in as much detail as one would like by some rule. ${ }^{2}$

The aim of this discussion note is to explain why Ormell's arguments should not convince philosophers that classical mathematics is wrong about the continuum. All that Ormell manages to show is that once the concept of a real number is restricted to include only those definable (and hence constructively acceptable), the set of such real numbers is denumerable (i.e., countably infinite). 'Definability' seems to be an intuitive informal notion, suggesting

1 C. Ormell, 'The Continuum: Russell's Moment of Candour', Philosophy 81, No. 4, (October 2006), 659-668.

2 Ormell, ibid, 663. A number is 'well-behaved' just in case it 'proceeds according to a law which enables us to calculate as many terms as we please'. Numbers are not well-behaved just in case (in writing out their decimal expansions) 'we can't write down their successive digits by following a rule of any sort'. In contemporary terms, well-behaved numbers are computable by some algorithm. Ormell says mathematics should admit only 'well-defined' numbers and considers 'well-behaved' numbers to be 'extremely well-defined'. 
that a number is definable just in case we can write something down that uniquely specifies that number. Thus, a number could be definable simply because we can write down all of the digits in its decimal expansion. However, the vast majority of numbers have infinite, nonrepeating decimal sequences. The only way that a finite mind can tame such an infinite sequence is by conceiving of it as governed by a rule. No one disputes that when the real numbers are restricted to definable numbers, there are only countably many. ${ }^{3}$ The core philosophical issue concerns whether all numbers must be definable.

In what follows, it will be useful to bear in mind the dialectic between constructivists and classical mathematicians. Classical mathematicians happily accept the inference:

(1) There are uncountably many real numbers.

(2) There are at most countably many definable numbers.

(3) Therefore, not all numbers are definable. ${ }^{4}$

Constructivists reject step (3) of the argument, because they hold that properly understood, all real numbers are definable. The general inference (from (1) and (2) to (3)) is valid. Furthermore, no one disputes (2). Thus, in order to reject the argument, constructivists have to reject premise (1). The grounds for rejecting (1) are that, once it is accepted that all numbers are definable, there can only be countably many reals. ${ }^{5}$

We contend, however, that the restriction of real numbers to those constructively definable is based on an unacceptable idealism, which regards the existence of numbers as dependent on some act of human minds. Only from this idealist perspective does it make sense to doubt

3 Indeed, since definable numbers must be defined using countably many symbols, the countability of definable numbers follows immediately.

One could further infer from (3) using classical logic that

(4) There exist indefinable real numbers.

Some constructivists would also reject the inference to step (4) from (3), because the transition

$$
\neg \forall \mathrm{x} F \mathrm{x} \rightarrow \exists \mathrm{x} \neg \mathrm{Fx}_{\mathrm{x}}
$$

is not valid in intuitionist logic. However, even constructivists who maintain classical logic will want to reject the argument on the basis of step (3).

5 One person's modus ponens is another's modus tollens tollens. That is, classical mathematicians argue that because reals are uncountable (as established by the diagonal argument), there must be indefinables. The constructivists argue that because there could not be indefinable numbers (given their philosophical conception of numbers), therefore the reals are countable. 


\section{On the Reality of the Continuum}

that the indefinable real numbers are proper objects of mathematical study. This idealist tendency is reflected in Wittgenstein's criticism of Cantor's diagonal argument, which is one of the original sources (aside from Brouwer) of the constructivist view Ormell advances. We further suggest that such idealism rests on a major mistake: that of mistaking epistemic limitations for metaphysical deficiencies. ${ }^{6}$ But, there are more things than are dreamt of in constructivist philosophy. To demand that what could be in the world is restricted to what we can dream of is to take a megalomaniacally anthropocentric view of human capacity in relation to the world.

\section{Of Rules and Reals}

Recall that Cantor reasoned as follows. If the real numbers were merely countably infinite, then it would be possible to enumerate them. However, if we draw up a list of reals (supposing we can do so) and pair each number of the list with a natural number, we immediately see that the list is incomplete. For we can always generate a real number not in the list by applying the diagonal procedure. For convenience we attempt to list just the real numbers between 0 and 1 , and we use the decimal representations of real numbers. Part of our list might look like this:

$0 . a_{1} a_{2} a_{3} a_{4} a_{5} a_{6} \ldots$

Natural numbers

$0 . b_{1} b_{2} b_{3} b_{4} b_{5} b_{6} \ldots$

1

0.c $\mathrm{c}_{1} \mathrm{c}_{2} \mathrm{c}_{3} \mathrm{c}_{4} \mathrm{c}_{5} \mathrm{c}_{6} \ldots$

$0 . \mathrm{d}_{1} \mathrm{~d}_{2} \mathrm{~d}_{3} \mathrm{~d}_{4} \mathrm{~d}_{5} \mathrm{~d}_{6}$...

$0 . \mathrm{e}_{1} \mathrm{e}_{2} \mathrm{e}_{3} \mathrm{e}_{4} \mathrm{e}_{5} \mathrm{e}_{6} \ldots$

4

$0 . f_{1} f_{2} f_{3} f_{4} f_{5} f_{6} \ldots$

5

$\ldots$ and so on.

To construct a number not on the list, we let $\delta=0 . \delta_{1} \delta_{2} \delta_{3} \delta_{4} \delta_{5} \delta_{6} \ldots$, where each $\delta_{\mathrm{i}}$ is different from the nth number in the nth row in our list. In each case, a number generated by the diagonal procedure is constructively well-defined, since it is given by a rule that we can readily specify and apply. Thus far, Cantor's diagonal argument is constructively impeccable. However, Cantor went on to suggest that, despite our not being able to list 'all the reals', we can still

6 On the charge of idealism leading philosophers astray when contemplating the continuum, see J. Franklin, 'Achievements and fallacies in Hume's account of infinite divisibility', Hume Studies 20 (1994), 85-101. 
think of the reals as a set with a definite size $\left(\aleph_{1}\right)$, a size greater than the number of natural numbers $\left(\aleph_{0}\right)$.

Many constructivists object to Cantor's conclusion at this point on the grounds that it seems to involve treating the real numbers as though they could form a completed whole. ${ }^{7}$ In particular, to give the totality of real numbers a definite cardinal number presupposes that the collection can be treated as a mathematical object in the same way that the finite numbers can. Indeed, this aspect of Cantor's approach is somewhat perversely called 'finitism' by Hallett [1984], who wishes to make the point that Cantor proposed to treat the infinite numbers as complete, definite, manipulable and susceptible to mathematical operations in roughly the same way as the finite numbers. ${ }^{8}$ But for constructivists to treat the infinite on par with the finite is to misconceive the nature of the former. As Dummett explains,

From an intuitionistic standpoint, the platonistic conception is the result of blatantly transferring, from the finite case to the infinite one, a picture appropriate only to the former. In making this transference, the platonist destroys the whole essence of infinity, which lies in the conception of a structure which is always in growth, precisely because the process of construction is never completed. ${ }^{9}$

However, it is clear this objection is far too fundamental to be the reason why constructivists such as Ormell baulk at the diagonal argument. If the problem is that it is illegitimate to gather the reals into a whole, because this operation destroys the potentially infinite nature of the reals, then this objection must apply also to the treatment of the natural numbers as a whole. Ormell appears sympathetic to this objection. He countenances countably infinite classes of numbers, but stops short of talking of countably infinite sets. However, strategically, the objection is a non-starter. If the goal is to convince classical mathematicians that Cantor's diagonal argument is wrong, it would not be wise to start by arguing for finitism out of metaphysical conviction.

7 The objection is common to Peirce, Brouwer, and Wittgenstein in their reactions to Cantor's argument.

8 M. Hallett, Cantorian Set Theory and Limitation of Size (Oxford: Oxford Logic Guides, 1994), 7.

9 M. Dummett, Elements of Intuitionism (Oxford: Clarendon Press, 1977), 57. (Ormell makes it clear that he is not an intuitionist, but as with Wittgenstein, he does share some of the early Brouwer's views.) 


\section{On the Reality of the Continuum}

Ormell's stated objection to the diagonal argument is different from the finitist scruples mentioned above. Ormell is concerned that the diagonal procedure will not generate all the reals needed to get an uncountably infinite set. There are only countably many reals generated by the diagonal procedure. The number of reals on the original list is also countable. Moreover, the union of countably many countably infinite sets is still countable. ${ }^{10}$ So merely adding the diagonal numbers to our list will not yield the uncountably infinite set of real numbers. To attain that result, we must add the indefinables.

What reason is there to believe in the existence of indefinables? Admittedly, the indefinables can seem a bit like the 'dark matter' of mathematics. Indefinables are needed to make the classical theory of the continuum come out right. The classical theory says there are $2^{\aleph_{0}}$ many real numbers between 0 and 1 , whereas each 'known' family of reals has at most $\aleph_{0}$ members. In the same way, physicists posit 'dark matter' in order to explain why so much less mass of matter in the universe is observed than is predicted by their best cosmological theory. 'Dark matter' cannot be detected in the same way as luminous matter, so the evidence for it is indirect. Similarly, there is no direct experiential evidence for the indefinables. Ormell says the indefinables are 'invisible, unproducable, and unbelievable'. ${ }^{11}$ Next Ormell might be expected to complain that the objects of mathematical analysis do not smell. This is a respect in which his empiricist idealism goes too far. ${ }^{12}$

More soberly, Ormell's main objection is that the idea of indefinables 'cannot survive the simple challenge, 'Well, let's examine one!". ${ }^{13}$ Although we cannot, of course, define an indefinable, we can describe how a family of such indefinables could be generated. A common way of doing so is to consider the number that might be produced by a particular fair coin tossing process. Every number

10 This result is sometimes proved using the Axiom of Choice which is not constructively acceptable, but it can be proved using countable choice.

11 Ormell, ibid, p. 667.

12 To lay our cards on the table, we are sympathetic to empiricism, but only of a realist (Aristotelian) variety. We hold that some very basic mathematical properties such as symmetry and ratios may be perceptible. We think that there could be real physical continua with the structure of the continuum, although it is an open question. We are sympathetic to the 'Aristotelian account' outlined in D. Gillies, 'An Empiricist Philosophy of Mathematics and its Implications for the History of Mathematics', The Growth of Mathematical Knowledge, E. Grosz and H. Breger, (eds.), 46-51.

13 Ormell, ibid, p. 664. 
can be represented in binary notation as a string of 0 s and $1 \mathrm{~s}$. We can allow ' 1 ' to correspond to a 'heads' result and ' 0 ' to correspond to a 'tails' result in coin tossing. Suppose that we start some particular process (which we can date and locate) and obtain the following initial digits for a number $\mathrm{R}^{*}$ :

$$
\mathrm{R}^{*}=0.111111001001001111100100111 \ldots
$$

What we have written down is not the number $\mathrm{R}^{*}$, but an initial segment of the number $\mathrm{R}^{*}$ and many other numbers sharing the same initial segment. If (let's say) the process generating $\mathrm{R}^{*}$ is random and never ending, then $\mathrm{R}^{*}$ is itself indefinable. There are limitations on our knowledge about $\mathrm{R}^{*}$. We cannot find out as much as we like. We cannot predict, for example, what the billionth digit in $\mathrm{R}^{*}$ is, unless, we are lucky enough to live to see the result. Nonetheless, it does seem that we can-in a manner of speaking-examine $\mathrm{R}^{*}$ even if only as a member of an equivalence class.

Ormell's view must be that we do not really know which object $\mathrm{R}^{*}$ is, since we cannot single it out directly in isolation. That is, we refer to $\mathrm{R}^{*}$, but only as a member of a totality of which it is a part. This appears to be an instance of impredicative definition. Constructivists find impredicative definitions objectionable, and claim that they are viciously circular. Frank Ramsey pointed out that impredicative definitions can be perfectly acceptable. We can define a certain man as 'the tallest member of his class', and there is nothing amiss with this impredicative definition. It is not obvious that impredicative definitions should not be acceptable when dealing with infinite totalities too. ${ }^{14}$

Ormell is right about one point, however. It is impossible to single out $\mathrm{R}^{*}$ in isolation. There is no rule that fits $\mathrm{R}^{*}$. Even if $\mathrm{R}^{*}$ exhibited some regularity for a while, there would be no guarantee that it would continue. It might repeat its initial segment a billion times before throwing up a different segment and then resume a repetition of its initial segment ad infinitum. Given the possibility of a number's divergence from expectations at some further point given an initial segment of the number's decimal expansion, it is impossible to know exactly which real number we are considering in the absence of a complete specification (or

14 M. Potter, Set Theory and its Philosophy (Oxford: Oxford University Press, 2004), 137. On the acceptability of impredicative definitions for classes, see M. Giaquinto, The Search for Certainty, (Oxford: Oxford University Press, 2002), 107. 


\section{On the Reality of the Continuum}

definition) of that number. ${ }^{15}$ Such a specification would consist either in an exact name for the object (as is easily provided for rational numbers, integers, special numbers like $\pi$ and e, and some others) or else in the specification of a rule for calculating each successive digit in the decimal expansion of the number. ${ }^{16}$ If we do not know exactly which number we are dealing with, then it will be difficult to say anything precise about it. The constructivist conception of mathematics prohibits acceptance of such unknown and perhaps unknowable entities. For example, Ormell says 'random sequences, if they exist out there, are not cashable as 'mathematical objects'. How could they be? Mathematics is the study of the implications of well-defined formal concepts'. ${ }^{17}$

\section{Idealism and Realism}

We think that the philosophical perspective motivating the constructivist restriction on the reals is fallacious. Once the rhetorical flourishes are expunged, the constructivist position amounts to the following:

Indefinable real numbers cannot exist, because they are not definitely conceivable. They are not surveyable and calculable by finite human minds.

Thus laid bare, this style of reasoning is fallacious, because we may lack an ability to definitely conceive of an entity $\mathrm{x}$ and yet it may still be the case that $\mathrm{x}$ is nonetheless real and, a fortiori, possible. European naturalists could not definitely conceive that there could be such a mammal as the platypus, and yet platypuses did exist while undiscovered by the European imagination.

Have we been unfair to constructivists? To be sure, constructivists do not deny the existence of indefinable reals outright. They deny the legitimacy or admissibility of such objects for mathematical study. It would clearly be a fallacy to move from the fact that we cannot define a number to the conclusion that such a number does not

15 This kind of reasoning is very close to L.Wittgenstein, Philosophical Grammar, (Oxford: Blackwell, 1974), p. 477. Here Wittgenstein states that all we have done is marked out an interval rather than closed in on a particular real number.

16 Cantor's diagonal technique is one such rule for constructing a welldefined real number.

17 Ormell, ibid, p. 664. 
exist. Rather, applying Occam's Razor, constructivists say that such indefinables should not figure in a mathematical theory. Taking a derogatory view of metaphysics and religion, constructivists are likely to suggest that indefinables may figure in these fields of study. ${ }^{18}$ Perhaps the requirement for 'admission' into non-mathematical fields of study is lower than the standard required for admission into mathematics!

But, wait, what is this talk of 'admissibility' really? The concepts of admissibility and legitimacy are allied to the notions of human authority and law. From the realist standpoint, numbers and other real things do not need admitting or legitimating by humans to come into existence. Furthermore, real objects are always legitimate objects of study in the sciences, even if they are not fully understood or known.

The following analogy will convey the realist assessment of the debate between constructivists and realists. Consider a field like astronomy, about which most people are realists. That is, they hold planets and stars really exist, and do so independently of what human beings think about them. Imagine some radical astronomers deciding that planets outside our solar system were 'inadmissible', because they could not be observed by current telescopes in such a way as to produce images of sufficiently high resolution. Suppose that a dispute then arises between the traditional and radical astronomers concerning the number of planets in the universe. The traditional astronomers claim that there are far more planets in the universe than nine, because they count the planets that are, from the radical perspective, 'inadmissible'. ${ }^{19}$ From a realist perspective, this is what the debate between constructivist and realists looks like.

From the constructivist perspective, of course, the story is not apt. Constructivists do not think that numbers have an existence independent of the minds that perceive them, in the way that planets exist independently of human observers. To them, the realist fallacy is to assume that mathematical theorems describe some fantastic landscape. As Wittgenstein put it in his critique of the diagonal argument,

The dangerous, deceptive thing about the idea: "The real numbers cannot be arranged in a series", or again "The set...is

18 Cf. N.Wildberger, 'Set Theory: Should You Believe?', http://web. maths.unsw.edu.au/ norman/papers/SetTheory.pdf, April 2006.

19 We prescind from the current debate over whether there are really eight or nine planets. 


\section{On the Reality of the Continuum}

not denumerable" is that it makes the determination of a concept-concept formation-look like a fact of nature. ${ }^{20}$

For constructivists, numbers must be regarded as human mental constructions. The motivation for so regarding them is to enable and ensure perfect knowledge of the numbers. According to one version of the verum-factum principle, we understand best what we ourselves produce. If all numbers are constructed by us, then presumably we will know all of their properties. Such a philosophical view cannot tolerate indefinables, the properties of which are not completely known. Realism, on the contrary, always brings with it the possibility of scepticism and incomplete knowledge.

Constructivists are sceptical about the existence of indefinable real numbers. In a revealing footnote, Ormell says:

They [indefinable real numbers] never feature in mathematical seminars, lectures, or conferences: they are never seen in mathematical textbooks or monographs. Their only role, it appears, is as fantasy objects which, it is hoped, might nominally save the official interpretation of the Diagonal Argument. ${ }^{21}$

Consequently, since we cannot know these numbers intimately, we should-by constructivists' lights-assume that they do not exist, or more cautiously, that they are 'inadmissible'.

Of course it is patently untrue that the indefinable real numbers never figure in mathematical seminars. What is true is that they do so as ensemble-players, as members of the continuum, rather than as individual lead actors (as independently specified decimals). Moreover, it is patently untrue that the only role of the indefinables is to save the diagonal argument. Without the indefinable reals, we do not have a complete field of real numbers. That is, we need such indefinables to 'fill in the gaps' between the points in the sparse constructive continuum. The important least upper bound property of continuous intervals requires indefinable reals, as does the seemingly obvious Intermediate Value Theorem (IVT). IVT simply says that if we have a function $f(x)$ that is continuous over the interval [a,b], and the value of the function at one endpoint is negative and at the other endpoint is positive (e.g. $f(b)>0$ and $f(a)<0)$, then the function must be equal to zero at some point $\mathrm{c}$ intermediate between $\mathrm{a}$ and $\mathrm{b}$

20 L. Wittgenstein, Remarks on the Foundations of Mathematics, ed. G.H. von Wright, R. Rhees, and GEM Anscombe, (Oxford: Blackwell, 1978), §19, .131.

21 Ormell, ibid, p. 664 fn. 9. 
(i.e. $f(c)=0$ for some $c$ in $(a, b))$. The analysis that results without these properties is difficult and different. ${ }^{22}$

\section{Uncomputable reals}

Liberal constructivism ought to allow the existence of uncomputable reals. It is not clear whether Ormell allows for such reals, because he does not discuss the distinction between computability and definability. The 'lawless decimals' he discusses are both indefinable and uncomputable. However, modern complexity theory allows for uncomputable, definable reals. Chaitin's construction $(\Omega)$ is a recipe for defining a number (called Chaitin's constant $\Omega_{\mathrm{L}}$ ) that gives the probability that a randomly chosen program in a given language L will halt. ${ }^{23}$ Chaitin's construction makes use of the fact that the halting problem-the problem of deciding whether an arbitrary programme in a language will terminate or continue endlesslyis undecidable (that is, there is no algorithm to compute the answer) for all programmes. The computation of Chaitin's constant would require solving the halting problem, which is impossible. So in each case - in each language L - Chaitin's constant is uncomputable. Nonetheless, Chaitin's constant is a particular probability, that is, a proportion in a set, a particular real number between 0 and 1 . It appears to be well-defined in the sense demanded by Ormell, of being fully describable in a language. It is possible to approximately calculate Chaitin's number in each case. So there is nothing ineffable or indefinable about such a number. The existence of uncomputable definable reals suggests the untenability of strict constructivism ('the algorithmic standpoint'), which admits as legitimate mathematical entities only those entities which can be computed by an algorithm. One of the most promising interpretations of Wittgenstein's philosophy of mathematics suggests that Wittgenstein was a strict constructivist. ${ }^{24}$ So much the worse for Wittgenstein and his friends in mathematics.

22 Eric Schechter, 'Constructivism is Difficult', The American Mathematical Monthly, 108, No. 1, January 2001, 50-54.

23 Gregory Chaitin, 'The limits of reason', Scientific American 294, No. 3 (March, 2006), 74-81.

24 On the interpretation of Wittgenstein as a proponent of 'the algorithmic standpoint', see M. Marion, Wittgenstein, Finitism, and the Foundations of Mathematics (Oxford: Clarendon Press, 1998). 


\section{On the Reality of the Continuum}

\section{Conclusion}

Thus, all that Ormell's discussion establishes is that the constructively acceptable real numbers (the definable reals) are countably infinite. This result has been known for a long time, so the primary interest in Ormell's discussion lies in his attempt to raise sceptical doubts about the existence of indefinable and random real numbers. However, as we have shown, his doubts about indefinables stem from an underlying empiricist idealism about the numbers that we find fallacious. Such idealism rests on the old Berkeleyan error: esse est percipi. We think that such idealism is no more applicable to the numbers than it is to tables and chairs. From a realist perspective, a lack of complete knowledge of the properties of numbers does not signal a lack of real existence.

Classical mathematics is doubtless committed to a whole hierarchy of infinities. Working mathematicians in the areas of set theory, analysis, and topology, are generally happy with this state of affairs. 'No one shall drive us out of the paradise which Cantor has created', Hilbert famously proclaimed at a mathematical congress in 1925. ${ }^{25}$ Wittgenstein replied that Cantorian mathematics was not a paradise, but a joke. ${ }^{26}$ We have argued that there is a philosophical confusion underlying the constructivist position: the conflation of what is real with what is epistemically conceivable. If this is right, then the joke is on Wittgenstein and his followers. The 'real' real numbers remain uncountably infinite.

University of New South Wales

25 D. Hilbert, 'Über das Unendliche', translated by E. Putnam and G.J. Massey as 'On the Infinite' in H. Putnam and P. Benacerraf, eds., Philosophy of Mathematics (Cambridge: Cambridge University Press, 1983), 191.

26 L. Wittgenstein, op.cit., V. 7, p. 264. 